\title{
Anterior chamber flare after trabeculectomy and after phacoemulsification
}

Institute of

Ophthalmology,

London EC1V 9EL, UK

D Siriwardena

A Kotecha

D Minassian

P T Khaw

Moorfields Eye

Hospital, London

EC1V 2PD

J K G Dart

Correspondence to:

Dr D Siriwardena, Wound

Healing and Glaucoma

Research Units, Institute of Ophthalmology and

Moorfields Eye Hospital,

11-43 Bath Street, London

EC1V 9EL

d.siriwardena@ucl.ac.uk

Accepted 26 April 2000

\author{
D Siriwardena, A Kotecha, D Minassian, J K G Dart, P T Khaw
}

\begin{abstract}
Aims-To evaluate and compare prospectively the anterior chamber inflammatory response after phacoemulsification cataract surgery and after trabeculectomy with peripheral iridectomy.

Methods-Anterior chamber inflammation was measured using the Kowa FM500 laser flare meter in 131 patients undergoing trabeculectomy and 148 patients undergoing phacoemulsification cataract extraction with intraocular lens implantation. Flare was measured before surgery and on each postoperative visit up to 12 months.

Results-Before surgery there was no significant difference in flare readings between the two groups. Following trabeculectomy flare returned to baseline levels 4 weeks after surgery, while following phacoemulsification cataract extraction it remained significantly higher at week 6 $(\mathrm{p}<0.006)$ and month $3(\mathrm{p}<0.05)$.

Conclusions-Anterior chamber inflammation is more prolonged after cataract surgery than after trabeculectomy. This may have implications for the timing of trabeculectomy in relation to cataract surgery.

(Br f Ophthalmol 2000;84:1056-1057)
\end{abstract}

Quantification of changes in the bloodaqueous barrier helps our understanding of the effects of anterior segment surgery and its complications. The purpose of this study was to evaluate and compare prospectively anterior chamber flare after phacoemulsification cataract extraction and after trabeculectomy with peripheral iridectomy.

Materials and methods

One hundred and thirty one consecutive patients undergoing routine trabeculectomy for primary open angle glaucoma were recruited. These patients had no history of pseudoexfoliation, uveitis, or previous ocular surgery. All trabeculectomies were performed as part of the ongoing (unmasked) Medical Research Council trial of intraoperative 5-fluorouracil. Approximately half received 5 -fluorouracil $(50 \mathrm{mg} / \mathrm{ml})$ and half received normal saline placebo, both being applied for 5 minutes on a sponge during the operation. All cases had a limbal based conjunctival flap. Postoperative management included suture release, ocular massage, and bleb needling. Seven patients received subconjunctival 5-fluorouracil injections.
One hundred and forty eight patients undergoing routine phacoemulsification surgery were also recruited. Their sole cause of visual loss was age related cataract and they had no other eye disease and no pseudoexfoliation. All phacoemulsification procedures were performed via a $3.2 \mathrm{~mm}$ clear corneal incision and completed with a $6 \mathrm{~mm}$ foldable silicon intraocular lens (Allergan S130NB). Viscoelastic (Provisc) was used during surgery and removed at the end of the procedure. A number of experienced surgeons performed all these operations at Moorfields Eye Hospital.

In patients undergoing trabeculectomy topical steroids (dexamethasone $0.1 \%$ or prednisolone acetate $1 \%$ ) were instilled 4-8 times daily for 6 weeks and then tapered off. Topical dexamethasone $0.1 \%$ was instilled $4-6$ times daily in the cataract patients for at least 4 weeks. Both groups of patients received topical chloramphenicol $0.5 \%$ four times daily for 1 month. Anterior chamber inflammation was measured before surgery and on each postoperative visit up to 12 months in all patients using the same Kowa FM-500 laser flare meter. This machine was calibrated regularly every 4 weeks. All flare readings were taken after pupil dilatation with tropicamide eye drops $1 \%$. On each occasion seven readings with a variation of less than $15 \%$ between background readings were taken; the two extreme values were discarded and the resulting mean and standard deviation calculated. Patients with significant surgical complications such as vitreous loss were excluded to prevent skewing of the data. A general linear model with logistic regression was used for statistical analysis (General Factorial Analysis from SPSS). The preoperative flare measurement was used as a covariate in this model. Data are expressed as mean (SE).

\section{Results}

The 131 patients who underwent trabeculectomy had a mean age of $69(0.7)$ years and $61.5 \%$ were male. The 148 patients who underwent cataract extraction had a mean age of $72(0.8)$ years and $42.9 \%$ were male. All patients were white and were not diabetic. Before surgery there was no significant difference in flare measurements between the two groups. Baseline flare in the trabeculectomy group was $9.4(0.7)$ photons/millisecond ( $\mathrm{ph} /$ $\mathrm{ms}$ ) and in the phacoemulsification group was $10.3(0.4) \mathrm{ph} / \mathrm{ms}$. Following trabeculectomy flare was highest 1 week after surgery at 25.0 (3.4) $\mathrm{ph} / \mathrm{ms}$ and returned to baseline levels by 4 weeks (Fig 1). Flare readings following phacoemulsification reached similar levels ini- 


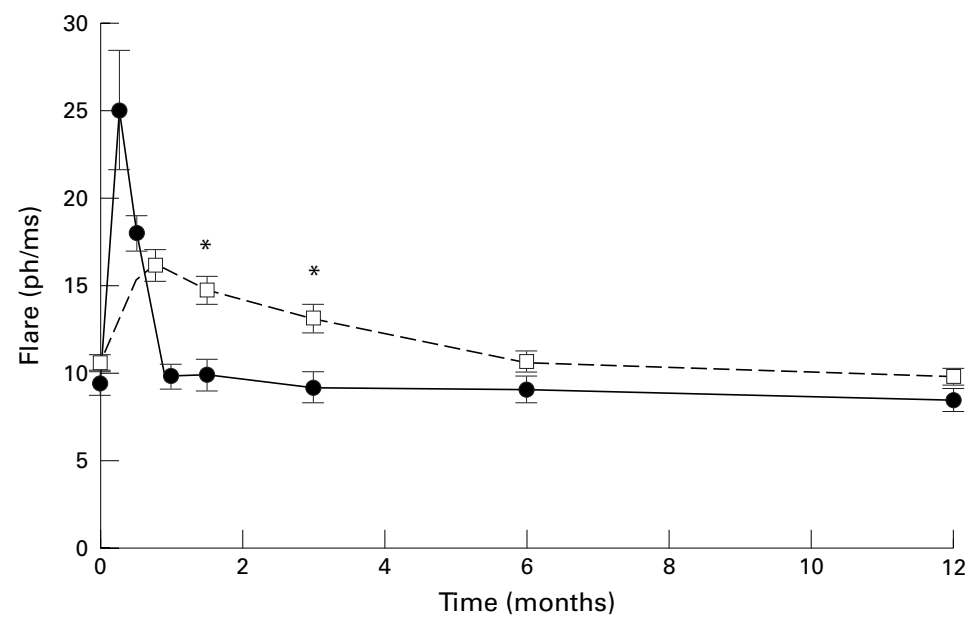

Figure 1 Laser flare before surgery and at postoperative visits up to 12 months in 131 trabeculectomy cases ( $\bullet$ ) and 148 phacoemulsification cataract extraction cases ( $\square)$. There was a significant difference between the two groups at week 6 and month $3\left({ }^{*} p<0.05\right)$.

tially (15.2 (0.6) $\mathrm{ph} / \mathrm{ms}$ at 3 weeks) but, in contrast to trabeculectomy, remained significantly higher at both week 6 (posttrabeculectomy flare $9.9(0.9) \mathrm{ph} / \mathrm{ms}$; postphacoemulsification flare $14.7(0.8) \mathrm{ph} / \mathrm{ms}$; $\mathrm{p}<0.006$ ) and at week 12 (post-trabeculectomy flare $9.2(0.9) \mathrm{ph} / \mathrm{ms}$; post-phacoemulsification flare $13.1(0.8) \mathrm{ph} / \mathrm{ms} ; \mathrm{p}<0.05)$. Flare in the phacoemulsification group returned to baseline levels by month 6 (post-trabeculectomy flare $9.1(0.7) \mathrm{ph} / \mathrm{ms}$; post-phacoemulsification flare $10.6(0.7) \mathrm{ph} / \mathrm{ms})$ and remained there, with no significant difference in flare readings between the two groups at 1 year (posttrabeculectomy flare $8.6(0.6) \mathrm{ph} / \mathrm{ms}$; postphacoemulsification flare $10.0(0.4) \mathrm{ph} / \mathrm{ms})$.

\section{Discussion}

Anterior chamber inflammation and breakdown of the blood-aqueous barrier is much more prolonged after uncomplicated small incision cataract surgery than after glaucoma filtration surgery with peripheral iridectomy. This prolonged low grade inflammation has been observed in other studies of phacoemulsification surgery ${ }^{1}$ but its significance may often have been underestimated. It is likely to be a product of the release of lens crystallins and lens epithelial cells into the aqueous humour, the effect of ultrasound, and/or the high volume of fluid passing through the eye at the time of surgery. These factors may upregulate the production of fibrogenic cytokines in the aqueous humour of patients who undergo phacoemulsification. ${ }^{23}$ These hypotheses remain to be tested, but our results indicate that such factors may be important in explaining why the success rate of phaco-trabeculectomy is significantly lower than trabeculectomy alone. ${ }^{4-6}$ It may also help to explain why recent cataract surgery is a risk factor for failure of filtration surgery.

Funding: Supported by the Medical Research Council, UK Grant G9330070 Moorfields/MRC Intraoperative 5-FU Study Grant G9323041 MRC Collaborative Phako Trial.

Conflict of interest: no proprietary interests.

1 Oshika T, Tsuboi S, Yaguchi S, et al. Comparative study of intraocular lens implantation through 3.2 and $5.5 \mathrm{~mm}$ incisions. Ophthalmology 1994;101:1183-90.

2 Nishi O, Nishi K, Ohmoto Y. Synthesis of interleukin-1, interleukin-6, and basic fibroblast growth factor by human cataract lens epithelial cells. F Cataract Refract Surg 1996;22(Suppl 1):852-8.

3 Allen JB, Davidson MG, Nasisse MP, et al. The lens influences aqueous humor levels of transforming growth factor-beta 2. Graefes Arch Clin Exp Ophthalmol 1998;236: 305-11.

4 Shields MB. Another reevaluation of combined cataract and glaucoma surgery. Am $\mathcal{F}$ Ophthalmol 1993;115:806-11.

5 Park HJ, Weitzman M, Caprioli J. Temporal corneal phacoemulsification combined with superior trabeculectomy. A retrospective case-control study. Arch Ophthalmol 1997;115:318-23.

6 Naveh N, Kottass R, Glovinsky J, et al. The long-term effect on intraocular pressure of a procedure combining trabeculectomy and cataract surgery, as compared with trabeculectomy alone. Ophthalmic Surg 1990;21:339-45.

7 Fluorouracil Filtering Surgery Study Group. Five-year follow-up of the Fluorouracil Filtering Surgery study. Am $\mathcal{F}$
Ophthalmol 1996;121:349-66. 